\title{
El uso de la plataforma Moodle para el aprendizaje del curso de filosofía en estudiantes universitarios
}

The use of the Moodle platform for the learning of the philosophy course in university students

A utilização da plataforma Moodle para a aprendizagem do curso de filosofia em estudantes universitários

\section{ARTÍCULO GENERAL}

\section{Teresa Elva Loayza Lozano \\ tloayza@unfv.edu.pe \\ https://orcid.org/0000-0001-5649-3746}

Universidad Nacional Federico Villareal, Lima - Perú

Recibido 07 de Abril 2021 | Arbitrado y aceptado 19 de Junio 2021 | Publicado en 03 Setiembre 2021

\section{RESUMEN}

El siguiente estudio pretendió evidenciar que el uso de la plataforma Moodle incide en el rendimiento académico de los estudiantes universitarios. Para ello, se aplicó una investigación de diseño cuasi-experimental considerando un grupo de control y grupo experimental, constituidos por alumnos matriculados en la asignatura de Filosofía en la Facultad de Derecho de una Universidad Pública de Lima. En el grupo de control, los estudiantes desarrollaron la materia académica en forma tradicional; mientras que en el grupo experimental los estudiantes utilizaron la plataforma Moodle para el desarrollo de todas las actividades concernientes a la asignatura. Para el proceso de recolección de datos, se aplicó dos instrumentos en forma de pretest y postest dirigidos a ambos grupos. Los datos resultantes determinaron que, el caso del pretest no se halló diferencias significativas en ambos grupos; por lo contrario, en el postest los datos porcentuales determinaron que ambos grupos difieren significativamente entre sí, al haber obtenido un valor equivalente a $Z=11,17 \mathrm{p}<, 001$. En consecuencia, se determinó que el grupo experimental obtuvo mayores puntajes, evidenciando que el uso de la plataforma Moodle influye significativa y positivamente en el el proceso enseñanza aprendizaje del curso de filosofía.

Palabras clave: Plataforma Moodle, aprendizaje, logros de aprendizaje, nuevas tecnologías, TIC.

\section{ABSTRACT}

The following study aimed to show that the use of the Moodle platform affects the academic performance of university students. For this, a quasi-experimental design investigation was applied considering a control group and an experimental group, made up of students enrolled in the Philosophy subject at the Law School of a Public University of Lima. In the control group, the students developed the academic subject in a traditional way; while in the experimental group the students used the Moodle platform for the development of all the activities concerning the subject. For the data collection process, two instruments were applied in the form of a pre-test and a post-test aimed at both groups. The resulting data determined that, in the case of the pretest, no significant differences were found in both groups; On the contrary, in the post-test the percentage data determined that both groups differ significantly from each other, having obtained a value equivalent to $\mathrm{Z}=11.17 \mathrm{p}<.001$. Consequently, it was determined that the experimental group obtained higher scores, showing that the use of the Moodle platform significantly and positively influences the teaching-learning process of the philosophy course.

Keywords: Moodle platform, learning, learning achievements, new technologies, ICT.

\section{RESUMO}

O estudo a seguir teve como objetivo mostrar que o uso da plataforma Moodle afeta o desempenho acadêmico de estudantes universitários. Para tanto, foi aplicada uma investigação de delineamento quase experimental considerando um grupo controle e um grupo experimental, formado por alunos matriculados na disciplina de Filosofia da Faculdade de Direito de uma Universidade Pública de Lima. No grupo de controle, os alunos desenvolveram a matéria acadêmica de forma tradicional; enquanto no grupo experimental os alunos utilizaram a plataforma Moodle para o desenvolvimento de todas as atividades relativas à disciplina. Para o processo de coleta de dados, foram aplicados dois instrumentos na forma de pré-teste e pósteste voltado para os dois grupos. Os dados resultantes determinaram que, no caso do pré-teste, não foram encontradas diferenças significativas em ambos os grupos; Pelo contrário, no pós-teste os dados percentuais determinaram que ambos os grupos diferem significativamente entre si, tendo obtido um valor equivalente a $\mathrm{Z}=11,17 \mathrm{p}<0,001$. Consequentemente, constatou-se que o grupo experimental obteve escores mais elevados, mostrando que o uso da plataforma Moodle influencia significativa e positivamente no processo de ensinoaprendizagem do curso de filosofia.

Palavras-chave: plataforma Moodle, aprendizagem, resultados de aprendizagem, novas tecnologias, TIC. 


\section{Introducción}

Hoy en día, el uso de las nuevas tecnologías de la información y comunicación (TIC) se han convertido en herramientas protagonistas dentro de las sociedades modernas que ha permitido a los seres humanos desenvolverse en diversos ámbitos a nivel socioeconómico, cultural, político y, principalmente, a nivel educativo, ya que está enfocada al desarrollo de distintas capacidades y destrezas en materia de inteligencia social, creatividad o innovación

Las TIC han modificado las formas de interacción e intercomunicación de las personas, y cada uno de estos aspectos se desarrolla en forma diferente entre los nativos e inmigrantes digitales, afectando incluso los procesos pedagógicos (Jara y Prieto, 2018).

En esa línea de ideas, las TIC aplicadas en el ámbito educativo a nivel universitario, se han constituido en nuevos espacios para el desarrollo de los aprendizajes; sin embargo, requieren de un adecuado manejo que conlleven a garantizar la conformación de nuevas competencias necesarias para el proceso formativo. Por tal motivo, resulta fundamental que las instituciones universitarias promuevan, garanticen e incluyan la utilización de las TIC durante el desarrollo de las actividades académicas, de tal forma que la comunidad estudiantil y el profesorado accedan a estos recursos (García, Reyes y Godínez, 2017, p. 305).

A la fecha, las instituciones universitarias han venido experimentando modificaciones sustanciales y revolucionarias en las formas de impartir enseñanza, diferenciándose de las maneras tradicionales de formación académica que se hayan ofrecido durante todos sus siglos de existencia.

A través de la utilización de las plataformas virtuales como espacios para el desarrollo creativo del conocimiento, se coloca a los participantes dentro del proceso formativo, autónomo, reflexivo, crítico y colaborativo, teniendo a los estudiantes como actores protagónicos de su propio aprendizaje.

En ese sentido, la plataforma Moodle es un aplicativo web desarrollado como plataforma LMS (sistema de gestión de aprendizaje), compuesta por un conjunto de recursos, a través del cual los estudiantes y los docentes pueden conformar las 
denominadas comunidades de aprendizaje e interactuar públicamente y de manera segura utilizando un nombre de usuario y una contraseña personal (González, 2016).

Esta herramienta, inspirada en el modelo educativo constructivista, está conformada por un sistema factible, cuya administración resulta ser muy realizable, permitiendo su adaptación a la naturaleza y necesidades de los usuarios o entidades que la implementen lo que la hace ser bastante operable, además de eficiente.

De allí que se pueda desarrollar el proceso formativo teniendo un enfoque de aprendizaje b-Learning, el cual incorpora actividades online dentro del proceso enseñanza-aprendizaje tradicional y que, según diversas investigaciones, permiten a los estudiantes desarrollar su propio ritmo de aprendizaje y personalización, manejarse de manera autónoma y tener determinadas capacidades. Por ejemplo, Sabaduche (2015) demostró que el 74 \% de estudiantes señalaron que la utilización de recursos virtuales de la plataforma Moodle facilitó su aprendizaje de las asignaturas impartidas. Por otro lado, De la Rosa (2011) evidenció que a través del uso de esta herramienta, estudiantes universitarios mejoraron su rendimiento académico. Asimismo, Black (2017) quien demostró que la utilización de la plataforma Moodle durante el proceso de enseñanzaaprendizaje los niveles de conocimientos en los estudiantes se incrementaron, lo cual conllevó a que se encuentren más motivados para su autoaprendizaje e interacción en foros para el intercambio de ideas entorno a distintas temáticas y contenidos desarrollados a través de este entorno. También, Mamby (2011) pudo demostrar que la enseñanza de la Filosofía desarrollada a través de plataformas tecnológicas educativas es un proceso que puede realizarse sin inconvenientes.

En ese sentido, puede determinarse que la inclusión de las TIC dentro del esquema tradicional de enseñanza permite a la comunidad estudiantil y al profesorado llevar a cabo sus materias académicas acorde con los tiempos actuales, adaptándose a las particularidades de cada estudiante.

Es decir, al proceso de enseñanza-aprendizaje tradicional dentro del salón de clases al que se le incluye una tecnología basada en sistemas web, es conocida como implantación tecnológica, que combinadas dan como resultado un modelo de innovación y motivación para los estudiantes, que les permite realizar sus actividades académicas dentro y fuera de las aulas (Belanger y Jordan, 2000, p. 65). 
Es preciso mencionar que el aprendizaje consiste en un conjunto de acciones concatenadas que permiten la estructuración de los conocimientos y significados, los cuales son inherentes a cada individuo, logrando que cada uno de ellos tenga un enfoque distinto, el cual puede autorregular (De Corte, 2007, p. 22).

Desde este enfoque, puede entenderse que el aprendizaje consiste en un proceso mediante el cual las personas adquieren capacidades y destrezas teniendo como premisa nueva información así como sus conocimientos previos o adquiridos mediante sus vivencias, que a su vez, generan o modifican aspectos comportamentales y actitudinales en cada individuo. Consecuentemente, estos aprendizajes toman ventajas cuando se combinan o complementan con el uso de las TIC, ya que a través de estas herramientas es posible superar las brechas de espacio y tiempo.

Entonces, se abre la posibilidad de plantear el logro de aprendizaje, el cual está asociado al nivel cognoscitivo que se evidencia en ciertos ámbitos o materias, cuya medición está sujeta a factores como el nivel académico y la edad del sujeto (Márquez y Jiménez, 2014, p. 85). No obstante, los resultados de un escueto proceso de evaluación del aprendizaje logrado de los estudiantes no proporcionan por sí mismos la totalidad de los patrones necesarios que permitan realizar acciones orientadas a la optimización de la calidad educativa.

Es por esa razón que el logro de aprendizaje está asociado a una escala de medición para valorar el nivel que los estudiantes han alcanzado en los entornos educativos; por lo tanto, se establece como el eje céntrico de la educación. Sin embargo, para alcanzar un adecuado logro de aprendizaje, es necesaria la intervención de múltiples factores extrínsecos al individuo, como la calidad del desempeño docente, los entornos donde se desarrollan las actividades académicas, el entorno familiar, los programas educativos, etc.

Asimismo, intervienen otros elementos asociados a factores psicológicos e intrínsecos al sujeto, como el aspecto actitudinal hacia el desarrollo de la materia académica, sus capacidades intelectuales, sus particularidades inherentes, el autoconcepto, la motivación, entre otros factores.

A partir de aquí, aparecen algunas preguntas respecto del aprendizaje autónomo, los sistemas de tutorías, el desarrollo de recursos educativos virtuales, el 
aprovechamiento de los recursos de las tecnologías al momento de interactuar virtualmente, la sensación de desilusión a recibir clases de la manera monótona y tradicional, entre otros.

Por tal motivo, resulta necesario identificar algunos aspectos asociados al logro de aprendizajes conceptual, procedimental y actitudinal en los usuarios de las plataformas virtuales, ya que, si bien el objetivo principal de la enseñanza de la asignatura de filosofía exige estos logros de aprendizaje, esto constituye un desafío importante tanto para los docentes que siguen manteniendo los métodos tradicionales (pizarra, plumón), omitiendo que las personas de las nuevas generaciones utilizan recursos de estos tiempos (tecnología).

Además, resulta todo un reto para las instituciones universitarias donde sigue habiendo resistencia en la incorporación de las herramientas tecnológicas como parte del proceso formativo.

En ese sentido, surgió la necesidad de conocer qué aspectos de la asignatura de filosofía deben considerarse para alcanzar el logro de aprendizaje conceptual, procedimental y actitudinal de los estudiantes utilizando las plataformas educativas virtuales como Moodle y constituirse como una alternativa del proceso educativo para este objetivo e implantar los beneficios de la comunicación interactiva mediante las tutorías en línea.

Por tal motivo, a través del siguiente trabajo investigativo se pretende resolver esta problemática a través de un análisis, ensayando la aplicación la plataforma educativa tecnológica Moodle como recurso didáctico para el logro de aprendizaje en los estudiantes del curso de filosofía de una Universidad pública de Lima Metropolitana.

\section{Material y métodos}

El presente estudio reúne las condiciones metodológicas de una investigación cuantitativa. Asimismo, presenta un diseño experimental de nivel cuasi-experimental. Este tipo de estudios requiere trazar un plan o estrategia concebida para obtener la información deseada, bajo condiciones experimentales y de control (Hernández, Fernández y Baptista, 2014). 
La población de estudio estuvo constituida por la totalidad de 64 estudiantes universitarios de una Universidad pública de Lima metropolitana. Para el proceso de recolección de datos se construyó dos pruebas: Una de entrada (Pre-test) y una de salida (Post-test) que estuvieron referidas al aprendizaje del curso de filosofía. En ambas pruebas se consideró tres aspectos para su elaboración: conocimientos con contenidos teóricos, conocimientos con contenidos prácticos o procedimentales y finalmente conocimientos con contenidos actitudinales de la asignatura en mención.

Previamente a la prueba de hipótesis se realizaron los análisis de normalidad para conocer como estos distribuidos los datos y a partir de ello elegir los estadísticos correspondientes.

El análisis estadístico de los datos, previamente colectados, se efectuaron con el apoyo del programa estadístico computarizado SPSS versión 25

\section{Resultados}

\section{Tabla 1}

Prueba $Z$ de comparación de medias de la pre-prueba por grupo de estudio

\begin{tabular}{|c|c|c|c|c|c|c|}
\hline Grupos & & & & & & \\
\hline Variables & Exp & & & & Z & Sig. \\
\hline & $\mathrm{M}$ & $\mathrm{DE}$ & $\mathrm{M}$ & $\mathrm{DE}$ & & \\
\hline Pretest & 11,81 & 5,54 & 11,69 & 5,89 & ,087 & ,931 \\
\hline
\end{tabular}

En la tabla 1 se puede apreciar que los resultados informan que los grupos de estudio; experimental y de control, no difieren entre si respecto de la pre prueba de evaluación. Este resultado permite afirmar que los grupos están distribuidos de manera homogénea, lo que en general es un buen indicador para la investigación puesto que significa un buen control de las variables. 
Tabla 2

Prueba $Z$ de comparación de medias de las áreas de la pre-prueba por grupo de estudio

\begin{tabular}{|c|c|c|c|c|c|c|}
\hline Grupos & & & & & & \\
\hline Variables & Exp & & & & Z & Sig. \\
\hline & $\mathrm{M}$ & $\mathrm{DE}$ & $\mathrm{M}$ & $\mathrm{DE}$ & & \\
\hline Conceptual & 5,81 & 2,87 & 6,03 & 2,88 &,- 305 & ,762 \\
\hline Procedimental & 6,00 & 2,90 & 5,66 & 3,13 & ,456 & ,650 \\
\hline Actitudinal & 36,03 & 7,43 & 33,97 & 5,76 & 1,241 &, 219 \\
\hline
\end{tabular}

En la tabla 2 se puede apreciar que los resultados informan que los grupos de estudio; experimental y de control, no difieren entre si respecto de la pre prueba de evaluación. Este resultado permite afirmar que los grupos están distribuidos en sus contenidos, conceptual, procedimental y actitudinal, de manera homogénea, lo que en general es un buen indicador para la investigación puesto que significa un buen control de las variables.

Tabla 3

Prueba $Z$ de comparación de medias de la post prueba por grupo de estudio

\begin{tabular}{|c|c|c|c|c|c|c|}
\hline Grupos & & & & & & \\
\hline Variables & Exp & ntal & & & Z & Sig. \\
\hline & $\mathrm{M}$ & $\mathrm{DE}$ & $\mathrm{M}$ & $\mathrm{DE}$ & & \\
\hline Posttest & 15,93 & 2,27 & 10,81 & 1,26 & 11,17 &, $001 * * *$ \\
\hline
\end{tabular}

El análisis de las diferencias entre los alumnos de los grupos experimental y de control, indica que existen diferencias estadísticas significativas $(Z=11,17 \mathrm{p}<, 01)$, notándose que la mayor calificación corresponde al grupo Experimental $(\mathrm{M}=15,93)$ respecto del grupo de control $(\mathrm{M}=10,81)$, lo que significa que la utilización de la plataforma virtual Moodle ha influido significativa y positivamente en el aprendizaje de la asignatura de Filosofía por parte de los estudiantes de la Facultad de Derecho de la Universidad Nacional Federico Villarreal. 
Tabla 4

Prueba $Z$ de comparación de medias de las áreas de la post prueba por grupo de estudio

\begin{tabular}{|c|c|c|c|c|c|c|}
\hline Grupos & & & & & & \\
\hline \multirow[t]{2}{*}{ Variables } & Exp & ntal & & & \multirow[t]{2}{*}{ Z } & \multirow[t]{2}{*}{ Sig. } \\
\hline & $\mathrm{M}$ & $\mathrm{DE}$ & $\mathrm{M}$ & $\mathrm{DE}$ & & \\
\hline Conceptual & 7,90 & 1,38 & 5,50 &, 76 & 8,65 &, $001 * * *$ \\
\hline
\end{tabular}

El análisis de comparación entre los alumnos de los grupos experimental y de control, indica que existen diferencias estadísticas significativas respecto del caso de los contenidos conceptuales $(\mathrm{Z}=8,65 \mathrm{p}<, 001)$, notándose que la mayor calificación corresponde al grupo Experimental $(\mathrm{M}=7,90)$ respecto del grupo de control $(\mathrm{M}=$ $5,50)$.

\section{Tabla 5}

Prueba $Z$ de comparación de medias de las áreas de la post prueba por grupo de estudio

\begin{tabular}{|c|c|c|c|c|c|c|}
\hline Grupos & & & & & & \\
\hline \multirow[t]{2}{*}{ Variables } & \multicolumn{2}{|c|}{$\begin{array}{c}\text { Experimental } \\
\mathrm{N}=17\end{array}$} & \multicolumn{2}{|c|}{$\begin{array}{l}\text { Control } \\
\mathrm{N}=17\end{array}$} & \multirow[t]{2}{*}{$\mathrm{Z}$} & \multirow[t]{2}{*}{ Sig. } \\
\hline & $\mathrm{M}$ & $\mathrm{DE}$ & $\mathrm{M}$ & $\mathrm{DE}$ & & \\
\hline Procedimental & 8,03 & 1,33 & 5,31 & 1,00 & 9,24 &, $001 * * *$ \\
\hline
\end{tabular}

El análisis de comparación entre los alumnos de los grupos experimental y de control, indica que existen diferencias estadísticas significativas respecto del caso de los contenidos procedimentales $(Z=9,24 \mathrm{p}<, 001)$, notándose que la mayor calificación corresponde al grupo Experimental $(\mathrm{M}=8,03)$ respecto del grupo de control $(\mathrm{M}=$ $5,31)$. 
Tabla 25

Prueba $Z$ de comparación de medias de las áreas de la post prueba por grupo de estudio

\begin{tabular}{|c|c|c|c|c|c|c|}
\hline \multicolumn{7}{|c|}{ Grupos } \\
\hline Variables & \multicolumn{2}{|c|}{$\begin{array}{l}\text { Experimental } \\
\mathrm{N}=17\end{array}$} & \multicolumn{2}{|c|}{$\begin{array}{l}\text { Control } \\
\mathrm{N}=17\end{array}$} & \multirow[t]{2}{*}{ Z } & \multirow[t]{2}{*}{ Sig. } \\
\hline & $\mathrm{M}$ & $\mathrm{DE}$ & $\mathrm{M}$ & $\mathrm{DE}$ & & \\
\hline Procedimental & 47,41 & 1,19 & 36,03 & 4,95 & 12,65 &, $001 * * *$ \\
\hline
\end{tabular}

El análisis de comparación entre los alumnos de los grupos experimental y de control, indica que existen diferencias estadísticas significativas respecto del caso de los contenidos procedimentales $(Z=12,65 \mathrm{p}<, 001)$, notándose que la mayor calificación corresponde al grupo Experimental $(M=47,41)$ respecto del grupo de control $(M=$ $36,03)$.

\section{Discusión}

En principio se debe mencionar que los instrumentos elaborados han sido cuidadosamente verificados en sus niveles de validez y confiabilidad, no solo porque se siguieron los criterios técnicos que se demanda, sino también porque se utilizaron los procedimientos técnicos y estadísticos para asegurar sus niveles de validez y confiabilidad. Los resultados logrados confirman que los dos instrumentos de evaluación cumplen con las exigencias técnicas de validez y confiabilidad.

Respecto de la hipótesis general de investigación que se planteó: El uso de la plataforma educativa virtual Moodle influye significativamente en los logros de aprendizaje de la asignatura de Filosofía en los estudiantes de la Facultad de Derecho de la Universidad Nacional Federico Villarreal 2018, los resultados dan cuenta de que en el post test, los estudiantes que conforman el grupo experimental alcanzan puntajes más elevados que los estudiantes del grupo de control, lo que se traduce en la existencia de diferencias significativas $(Z=11,17 \mathrm{p}<, 001)$. A partir de este resultado se puede afirmar, no solo que la hipótesis general ha sido respaldada, sino también que el uso de la plataforma Moodle es una verdadera alternativa para incrementar significativamente los niveles de rendimiento de los estudiantes. 
Estos resultados parecen confirmar lo que pudieron lograr autores como Maldonado (2019) quien concluye que la aplicación de la plataforma Moodle mejora significativamente el aprendizaje de los estudiantes, pero también se puede apreciar que a ellos les complace recibir las clases on-line. Por su parte Aguirre (2019) sus resultados le permiten sugerir la utilización masiva de la plataforma Moodle en todas las universidades del país, por su parte Black (2017) concluye que la implementación de ambientes virtuales es importante en la educación, ya que fortalece el proceso de enseñanza - aprendizaje. Rojas (2017) prueba que el grupo experimental obtiene valores más altos que el grupo de control, cuando se utiliza la plataforma Moodle, por otra parte, Sabaduche (2015) prueba que los estudiantes consideran que las herramientas virtuales han facilitado su aprendizaje en el curso, finalmente Canseco (2013) muestra que el $98 \%$ de estudiantes consideran que el manejo del aula virtual es fácil, permitiendo al docente trabajar sin dificultades.

Tal vez lo novedoso que ha resultado el uso de la plataforma Moodle y que ha ubicado a los estudiantes en una realidad más cercana a la que habitualmente ellos viven, ha hecho que acepten rápidamente esta nueva forma de enseñar y aprender y la incorporen a su quehacer académico diario. Seguramente son esta las razones por las que los estudiantes asumen el cambio y modifican sus actitudes respecto del estudio y el aprendizaje, tal como se puede desprender de los resultados que se han obtenido en la presente investigación.

Por otra parte, los resultados logrados con el uso de la plataforma Moodle hace que la asignatura de Filosofía se ubique en un escenario más moderno, en la medida de que las plataformas virtuales son verdaderas herramientas que, utilizadas apropiadamente, son de mucha utilidad en tanto van a facilitar los procesos de comunicación entre los estudiantes y sus docentes. Adicionalmente y no menos importante, la utilización de la plataforma Moodle permite la realización de actividades como el foro, que llevan a que los estudiantes puedan participar de manera activa en los debates, exposiciones, por lo que se ven motivados a investigar, analizar las diversas propuestas teóricas de manera que estén mejor preparados para cumplir estas tareas.

Siguiendo a Bautista (2016), se debe afirmar que las nuevas tecnologías de la comunicación han llevado a plantearse ya como una necesidad de urgente solución, una reforma educativa de tal magnitud que modifique sustantivamente las formas 
pedagógicas de manera que se puedan adaptar a los nuevos tiempos y formar a los estudiantes de todos los niveles educativos como verdaderos ciudadanos, profesionales y personas con altos valores que contribuyan al desarrollo de la nación. Las nuevas metodologías pedagógicas pueden, tomando como base las nuevas tecnologías de la comunicación, implementarse en la actualidad con altos niveles de éxito y en los que el rol del docente y el propio estudiante deben ser replanteados.

Asimismo, se pudo establecer las siguientes conclusiones:

1. En lo que se refiere al pretest, los resultados estadísticos indican que no se encontraron diferencias significativas entre el grupo experimental y el de control, por lo que se concluye que ambos grupos son similares u homogéneos.

2. En el caso del post test los resultados estadísticos muestran que los grupos de investigación difieren significativamente entre sí $(Z=11,17 \mathrm{p}<, 001)$, siendo el grupo experimental quien alcanza los puntajes más altos, razón por la cual se concluye que la utilización de la plataforma Moodle es muy importante para el proceso enseñanza aprendizaje.

3. En el caso de los contenidos conceptuales del curso de Filosofía, los resultados estadísticos muestran que los grupos de investigación difieren significativamente entre sí $(Z=8,65 \mathrm{p}<, 001)$, siendo el grupo experimental quien alcanza los puntajes más altos.

4. En el caso de los contenidos procedimentales del curso de Filosofía, los resultados estadísticos muestran que los grupos de investigación difieren significativamente entre sí $(Z=9,24 p<, 001)$, siendo el grupo experimental quien alcanza los puntajes más altos.

5. En el caso de los contenidos actitudinales del curso de Filosofía, los resultados estadísticos muestran que los grupos de investigación difieren significativamente entre sí $(Z=12,65 p<, 001)$, siendo el grupo experimental quien alcanza los puntajes más altos

Consecuentemente, se planteó las siguientes recomendaciones:

Primero: El uso de plataformas como el Moodle debe ser utilizado en todas las instituciones educativas públicas de todos los niveles, de la básica a la superior, de manera que las actividades académicas se realicen en altos niveles de calidad. 
Segundo: Se considera, además, que el conjunto de profesores de nuestra Facultad debe estudiar más acerca de la Plataforma educativa Moodle para poder explotar al máximo las posibilidades que brinda y así poder organizar los cursos con la mayor calidad posible.

Tercera: La realidad impone que las universidades promuevan el uso de modalidades pedagógicas diversas, de manera que se facilite los procesos de aprendizaje de los estudiantes, así como los procesos de enseñanza por parte de los docentes. Se debe hacer énfasis en las estrategias provenientes de las nuevas tecnologías de la comunicación.

Cuarto: Se sugiere que las autoridades tomen acciones para modificar y equipar la infraestructura y los recursos disponibles en las aulas de clases de la Facultad de Derecho, de manera que los docentes puedan contar con los materiales y equipos necesarios para cumplir eficientemente con su labor académica.

Quinto: Se sugiere que, a nivel general, las autoridades responsables del sector educación, puedan desarrollar de manera masiva, cursos de capacitación para los docentes en la perspectiva de que puedan manejar eficientemente las herramientas tecnológicas de manera que se optimice su labor académica

\section{Agradecimiento}

Se agradece al Dr, Manuel Torres Valladares, asesor por sus recomendaciones, comentarios y aportaciones al desarrollo de este trabajo investigativo

\section{Referencia}

Aguirre, M. (2019). Uso de la plataforma Moodle y el desempeño académico de los estudiantes de la carrera de Electrónica Industrial en la asignatura de Matemática I, de la Escuela de Ingeniería en el Instituto Superior Tecnológico Privado CIBERTEC 2017-II. [Tesis de maestría]. Universidad Nacional Mayor de San Marcos.

Belanger, F., \& Jordan, D. (2000). Evaluation and Implementation of Distance Learning: technologies, tools and techniques. Idea Group Publishing. 
Black, S. (2017). Utilización de la plataforma MOODLE en la asignatura de Ciencias

Sociales - un estudio de caso en estudiantes de primero de bachillerato de la institución educativa “Lev Vygotsky”. [Tesis de maestría]. Escola Superior De Educação E Ciências Sociais.

De Corte, E. (2007). Learning from instruction: The case of mathematics. Learning Inquiry, 1, 19-30.

De la Rosa, J. (2011). Aplicación de la plataforma moodle para mejorar el rendimiento académico en la enseñanza de la asignatura de cultura de la calidad total en la Facultad de Administración de la Universidad del Callao. [Tesis doctoral]. Universidad Nacional Mayor de San Marcos.

García, M., Reyes, J., \& Godínez, G. (2017). Las Tic en la educación superior, innovaciones y retos. Revista Iberoamericana de las Ciencias Sociales y Humanisticas: RICSH, 6(12), 299-316. doi:10.23913/ricsh.v6i12.135

Gonzáles, L. (2016). Una breve historia de Moodle: Entrevista con la Mtra. Rebeca Valenzuela Argüelles. Revista Digital Universitaria, 17(8), 1-5.

González, C. (2012). Aplicación del Constructivismo Social en el Aula. Guatelama: Instituto para el Desarrollo y la Innovación Educativa en Educación Bilingüe y Multicultural -IDIE - Organización de Estados Iberoamericanos para la Educación la Ciencia y la Cultura -OEI- Oficina Guatemala.

Hernández, R., Fernández, C., \& Baptista, P. (2014). Metodología de la investigación (6ta ed.). McGraw Hill.

Jara, N., \& Prieto, C. (2018). Impacto de las diferencias entre nativos e inmigrantes digitales en la enseñanza en las ciencias de la salud: revisión sistemática. Revista Cubana de Información en Ciencias de la Salud, 29(1), 92-105.

Maldonado, E. (2019). Uso de la plataforma virtual Moddle y su influencia en el aprendizaje significativo en el área de tecnología e informática de los alumnos del curso octavo del Colegio Educativo Técnico Industrial del Municipio de Garagoa-Boyacá, 2018. [Tesis de maestría]. Universidad de San Martín de Porres. 
Mamby, J. (2011). La enseñanza de la filosofía en espacios virtuales. [Tesis de Maestría]. Universidad de San Buenaventura.

Rojas, I. (2017). Plataforma Moodle y su influencia en la actitud hacia el aprendizaje virtual en estudiantes de la facultad de estudios a distancia - Universidad pedagógica y tecnológica de Colombia, 2015. [Tesis doctoral]. Universidad Norbert Wiener.

Sabaduche, D. (2015). Herramientas virtuales orientadas a la optimización del aprendizaje participativo: Estado del Arte. Revista de Ciencias Empresariales de la Universidad de San Martín de Porres, 6(1), 12-23. 